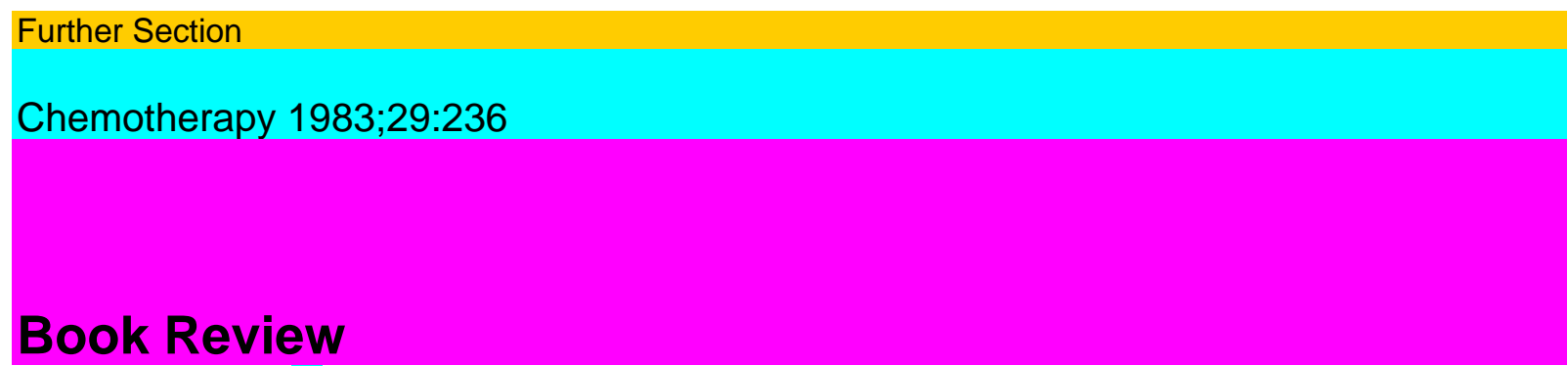

\title{
G. Delgado,J.P.Smith
}

Management of Complications in Gynecologic

Oncology

Wiley, Chichester 1982287 pp.; E29.75 ISBN 0-471-05993-5

Gynecologic oncology is a relatively new discipline which requires knowledge not only of the three major modalities of cancer treatment (surgery, radiotherapy, and chemotherapy), but also their complications. Existing texts on gynecologic oncology discuss diagnosis, staging and treatment of gynecologic cancers, but do not adequately cover complications of this treatment. Since these complications may involve a wide range of medical, surgical, radiotherap-eutic, and chemotherapy problems, several texts would need to be consulted. In addition, they may involve the gastrointestinal system and urinary tract as well as the genital tract. Prior to the publication of this text, the gynecologic oncologist did not have a single text that discussed this wide range of complications. This text will fill that void in gynecologic oncology.

The text begins with a thorough discussion of the exploration of the abdomen, then moves to the 'surgical complications', and ends with 'medical complications'.

The strongest and most complete section of the text is 'surgical complications'. Several wellknown gynecologic oncologists, a urologist, and a vascular surgeon joined the authors in this section to cover

such areas as: vascular complications, bowel complications (irradiated and non-irradiated), urologic complications (surgical and irradiation), complications of lymphadenectomy, and complications of vulva and vaginal surgery. This section is well written with excellent illustrations of the techniques and has a complete bibliography. The discussion includes newer techniques in gynecologic oncology including: arterial embolization for hemorrhage, automatic stapler instruments, and myocutaneous grafts. This section is particularly thorough in its discussion of radiation complications and the surgical techniques to correct these problems. This encompasses bowel, bladder, ureters, vagina, and vulva.

The section on medical complications includes chapters on venous thrombosis and pulmonary em-bolus, chemotherapy complications, infectious complications, and hyperalimentation. This section is far less complete than the previous section on surgery. Pulmonary complications are not covered well, and a discussion of respiratory management is not included. Medical evaluation of the patient prior to surgery is not discussed. Management of the critically ill patient in an intensive care environment is not discussed. These omissions reflect the interest of the authors which is primarily surgical.

In summary, the text is an excellent one for the surgically oriented specialist in gynecologic oncology who must repair radiotherapy and surgical complications. Its weakness is in medical complications and the management of the critically ill patient.

F.M. Schabel, Birmingham, Ala. 